\title{
Tuberculosis-Associated Secondary Pneumothorax: A Retrospective Study of 53 Patients
}

\author{
Masoud Shamaei MD, Payam Tabarsi MD, Saviz Pojhan MD, Leila Ghorbani, \\ Parvaneh Baghaei MD, Majid Marjani MD, and Mohammad Reza Masjedi MD
}

\begin{abstract}
BACKGROUND: Pneumothorax is a well known complication of pulmonary tuberculosis (TB), particularly in patients with advanced TB. METHODS: At our national TB-referral hospital, we compared the medical records of $53 \mathrm{~TB}$ patients with pneumothorax and $106 \mathrm{~TB}$ patients without pneumothorax, seen in 2003 to 2008 . We analyzed data on demographics; TB type (smear-positive, smear-negative, extrapulmonary); patient type (new patient, relapse, treatment default, treatment failure); clinical and radiological manifestations; surgeries; and outcomes. RESULTS: Of the 53 pneumothorax patients, 34 (64\%) were male. The pneumothorax group's mean age was $34 \mathrm{y}$ (range 14-76 y). Thirty-six $(68 \%)$ of the pneumothorax patients were new TB cases (ie, TB undiagnosed before they presented with pneumothorax). Pneumothorax was not significantly associated with sex, smoking, or drug use. Pneumothorax was significantly more common in patients < 30 years old $(P<.001)$. In terms of radiological manifestations, 20 pneumothorax patients $(38 \%)$ had cavitary lesions, and pulmonary infiltration and effusion were present in $19(36 \%)$ and $17(32 \%)$ patients, respectively. Cavitary lesion was significantly more common among the pneumothorax patients $(P=.006)$. Overall, $47(89 \%)$ of the pneumothorax patients were relieved with chest-tube insertion; the other pneumothorax patients were only observed. CONCLUSIONS: In patients < 30 years old or with cavitary lesions, worsening of the patient's respiratory condition should prompt consideration of pneumothorax. Key words: pulmonary tuberculosis; pneumothorax; surgery. [Respir Care 2011;56(3):298-302. (C) 2011 Daedalus Enterprises]
\end{abstract}

\section{Introduction}

An ancient infectious disease, tuberculosis (TB) has affected humankind throughout history. Despite the introduction of medical TB treatment during the 20th centu-

\footnotetext{
Masoud Shamaei MD, Payam Tabarsi MD, Leila Ghorbani, Parvaneh Baghaei MD, Majid Marjani MD, and Mohammad Reza Masjedi MD are affiliated with the Mycobacteriology Research Center; and Saviz Pojhan MD is affiliated with the Transplant Research Center, National Research Institute of Tuberculosis and Lung Disease, Masih Daneshvari University Hospital, Shahid Beheshti University of Medical Sciences, Tehran, Iran.

The authors have disclosed no conflicts of interest.

Correspondence: Masoud Shamaei MD, Mycobacteriology Research Center; National Research Institute of Tuberculosis and Lung Disease, Masih Daneshvari University Hospital, Shahid Beheshti University of Medical Sciences, Darabad, Niavaran Square, Tehran, 1955841452, Iran. E-mail: dr_shamaei@yahoo.com.
}

DOI: $10.4187 /$ respcare. 00695 ry, ${ }^{1,2}$ TB is the leading cause of mortality among curable infections. ${ }^{3,4}$ This problem becomes more evident when considering the increasing incidence of human immunodeficiency virus/acquired immune deficiency syndrome. ${ }^{4-6}$ These diseases have sped each other's progress, giving rise to the terms "co-epidemic" and "dual epidemic." The majority of deaths due to TB occur in developing countries, as is emphasized in the World Health Organization's 2006 report, "Global Tuberculosis Control: Surveillance, Planning, Financing." 7 In view of these issues, with the increasing incidence of TB and improving TB survival, the incidence of TB complications that need surgery has increased and merits more thorough consideration. ${ }^{8,9}$

Pneumothorax is one of the important TB complications that requires surgery. ${ }^{8-11}$ Pneumothorax secondary to TB usually occurs after extensive TB involvement of the lung, and the sudden onset of bronchopleural fistulization and empyema ${ }^{12}$ with severe cavitary formations or occasionally with miliary TB. The TB organism invades the pleura and causes liquifactive necrosis, then pleural rupture. ${ }^{12}$ 


\section{Tuberculosis-Associated Secondary Pneumothorax}

Therefore, a remaining apical lesion after lung re-expansion following a spontaneous pneumothorax should raise suspicion of pulmonary TB. ${ }^{12}$ Although re-expansion occurs in some patients due to TB treatment, most of these patients require chest-tube insertion, which is the treatment of choice for complete drainage and resolution. ${ }^{13,14}$

At Masih Daneshvari Hospital, Iran's sole TB-referral center and a specialized center for chest disease and thoracic surgery, we see numerous patients with TB-induced pneumothorax or hydropneumothorax. We evaluated patients with TB-induced pneumothorax or hydropneumothorax and assessed the outcomes of surgeries.

\section{Methods}

This was a descriptive study in which we retrospectively reviewed patient medical records at Masih Daneshvari Hospital. We reviewed the medical records of $53 \mathrm{~TB}$ patients with pneumothorax, and 106 TB patients without pneumothorax, seen March 21, 2003, to March 20, 2008. We analyzed data on demographics from the first in-patient visit, before treatment; TB type (smear-positive, smear-negative, extrapulmonary); patient type (new patient, relapse, treatment default, treatment failure); clinical and radiological manifestations; surgeries; and outcomes. The control group consisted of 106 patients hospitalized during the study period with confirmed active TB but who did not require surgery. We analyzed the data with statistics software (SPSS 11.5, SPSS, Chicago, Illinois). We used the chi-square test, the Fisher exact test, and the $t$ test, as appropriate. We considered a $P$ value of $<.05$ statistically significant.

TB diagnosis was based on a positive smear, positive culture of sputum for Mycobacterium tuberculosis, or tissue pathology consistent with TB. We considered patient type (new case, relapse, treatment failure, or multipledrug-resistant TB), and TB type (smear-positive, smearnegative, or extrapulmonary), according to World Health Organization definitions. ${ }^{15}$ We identified clinical TB manifestations from the patient's history, and radiological manifestations from the chest-radiographs and computed tomograms that are performed for all TB patients and interpreted by expert chest radiologists.

The scientific and ethics committee of Iran's National Research Institute of TB and Lung Disease approved the study.

\section{Results}

Table 1 describes the demographics of the 53 pneumothorax patients and the 106 non-pneumothorax patients. Table 2 describes the patient types, disease types, signs and symptoms, radiographic patterns, and pneumothorax patterns. The chi-square analysis indicated that pneumo-
Table 1. Demographics $(n=159)$

\begin{tabular}{lccc}
\hline \hline & $\begin{array}{c}\text { With } \\
\text { Pneumothorax } \\
\text { no. (\%) }\end{array}$ & $\begin{array}{c}\text { Without } \\
\text { Pneumothorax } \\
\text { no. (\%) }\end{array}$ & $P$ \\
\hline Male & $34(64)$ & $57(54)$ & .14 \\
Female & $19(36)$ & $49(46)$ & \\
Age & & & \\
$\quad<30$ y & $26(49)$ & $25(24)$ & $<.001$ \\
$\quad 30-60$ y & $22(42)$ & $40(38)$ & \\
$>60$ y & $5(9)$ & $41(39)$ & \\
Active smoker & $23(43)$ & $38(36)$ & .23 \\
Opium inhalation addiction & $18(34)$ & $25(24)$ & .12 \\
Close-contact history & $10(19)$ & $28(26)$ & .20 \\
Tuberculosis treatment history & $17(32)$ & $25(24)$ & .17 \\
Presented with pneumothorax & $22(42)$ & NA & NA \\
$\quad$ before tuberculosis & & & \\
$\quad$ diagnosed & & & \\
Total & 53 & 106 & NA \\
& & & \\
\hline NA = not applicable & & & \\
\hline
\end{tabular}

thorax was not significantly associated with sex, smoking, or illicit drug use. Conversely, age had normal distribution by the Kolmogorov-Smirnov test, and the $t$ test for independent samples indicated that pneumothorax was significantly associated with age $(P<.001)$. With the chi-square test, pneumothorax was significantly more common among patients $<30$ years old $(P<.001)$.

Of the 53 pneumothorax patients, $44(83 \%)$ were smearpositive. The most common symptoms were cough (85\%), dyspnea (70\%), and expectoration (70\%). As we expected, weight loss was significant $(P=.01)$. Twenty patients (38\%) had cavitary lesions, 19 (36\%) had pulmonary infiltration, and 17 (32\%) had effusion. Notably, the incidence of cavitary lesions was significantly higher among the pneumothorax patients $(P=.006)$. Other radiological findings were not significantly different (see Table 2).

The mean \pm SD interval between first TB treatment and pneumothorax was $16 \pm 47$ days (range $-22 \mathrm{~d}$ to $258 \mathrm{~d}$ ). Forty-seven patients (89\%) required surgery, and $6(11 \%)$ did not. Surprisingly, 22 (42\%) of the pneumothorax patients presented initially with pneumothorax, and their TB was diagnosed during hospitalization. Of the 47 patients who received surgery, all initially received a chest tube, and $40(85 \%)$ of those were relieved with chest-tube insertion alone. Thoracotomy was performed on 6 pneumothorax patients (13\%). One pneumothorax patient $(2 \%)$ underwent pneumonectomy. Of the 47 patients who received surgery, $6(13 \%)$ did not favorably respond to surgery and the lungs did not re-expand. In 27 patients (57\%) the median number of days the chest tube was in place was 13 days (interquartile range $8-25 \mathrm{~d}$ ), and the pneumothorax completely resolved. In the other 14 patients (30\%) the chest tube was changed to an empyema tube because of 


\section{Tuberculosis-Associated Secondary Pneumothorax}

Table 2. Patient Types, Disease Types, Signs and Symptoms, Radiographic Patterns, and Pneumothorax Patterns

\begin{tabular}{|c|c|c|c|}
\hline & $\begin{array}{c}\text { With } \\
\text { Pneumothorax } \\
\text { no. }(\%)\end{array}$ & $\begin{array}{c}\text { Without } \\
\text { Pneumothorax } \\
\text { no. }(\%)\end{array}$ & $P$ \\
\hline \multicolumn{4}{|l|}{ Patient Type } \\
\hline New patient & $36(68)$ & $67(63)$ & .38 \\
\hline Relapse & $8(15)$ & $10(9)$ & \\
\hline Treatment default & $2(4)$ & $10(9)$ & \\
\hline Treatment failure & $7(13)$ & $19(18)$ & \\
\hline \multicolumn{4}{|l|}{ Tuberculosis Type } \\
\hline Smear positive & $44(83)$ & $72(68)$ & .13 \\
\hline Smear negative & $4(8)$ & $14(13)$ & \\
\hline Extrapulmonary & $5(9)$ & $20(19)$ & \\
\hline \multicolumn{4}{|l|}{ Signs and Symptoms } \\
\hline Cough & $45(85)$ & $85(80)$ & .26 \\
\hline Sputum & $37(70)$ & $68(64)$ & .23 \\
\hline Hemoptysis & $8(15)$ & $17(16)$ & .54 \\
\hline Pleuritic chest pain & $31(57)$ & $46(43)$ & .08 \\
\hline Dyspnea & $37(70)$ & $76(72)$ & .47 \\
\hline Appetite loss & $17(32)$ & $32(30)$ & .47 \\
\hline Weight loss & $26(49)$ & $31(29)$ & .01 \\
\hline Sweating & $12(23)$ & $68(64)$ & .48 \\
\hline Fever & $36(68)$ & $68(64)$ & .39 \\
\hline \multicolumn{4}{|l|}{ Radiographic Pattern } \\
\hline Cavitation & $20(38)$ & $19(18)$ & .006 \\
\hline Infiltration & $19(36)$ & $42(40)$ & .39 \\
\hline Consolidation & $21(49)$ & $23(21)$ & .19 \\
\hline Calcification & $3(6)$ & $13(12)$ & .15 \\
\hline Effusion & $17(32)$ & $31(29)$ & .42 \\
\hline Pleural thickening & $12(23)$ & $23(21)$ & .58 \\
\hline Tree in bud & $3(6)$ & $8(8)$ & .47 \\
\hline Bronchiectasis & $9(17)$ & $32(30)$ & .37 \\
\hline Emphysema & $5(9)$ & $16(15)$ & .23 \\
\hline Collapse & $9(17)$ & $12(11)$ & .23 \\
\hline Fibrodestructive changes & $7(13)$ & $10(9)$ & .32 \\
\hline \multicolumn{4}{|l|}{ Pneumothorax Pattern } \\
\hline Right & $35(66)$ & NA & NA \\
\hline Left & $10(19)$ & NA & NA \\
\hline Bilateral & $8(15)$ & NA & NA \\
\hline Pneumohydrothorax & $18(34)$ & NA & NA \\
\hline Total & 53 & 106 & NA \\
\hline
\end{tabular}

empyema, lung destruction, bronchopleural fistula, or the patient was smear-positive but unable to undergo thoracotomy or pneumonectomy. Of the 6 patients who did not initially respond to surgery, 2 had recurrent empyema; they had thoracotomy/decortication and pneumonectomy, respectively. The other 3 patients had extensive lung destruction, known Langerhans cell histiocytosis (histiocytosis $\mathrm{X}$ ) and TB, and they died of recurrent pneumothorax: one after admission with severe respiratory distress and massive pneumothorax.
Table 3. Surgeries and Outcomes in Tuberculosis Patients With Pneumothorax

\begin{tabular}{lcc}
\hline \hline & $\begin{array}{c}\text { Intervention } \\
\text { Patients } \\
\text { no. }(\%)\end{array}$ & $\begin{array}{c}\text { Total } \\
\text { Patients } \\
\text { no. }(\%)\end{array}$ \\
\hline Surgical Intervention & & \\
$\quad$ Chest tube & $40(85)$ & $40(76)$ \\
Thoracotomy & $6(13)$ & $6(11)$ \\
Pneumonectomy & $1(2)$ & $1(2)$ \\
$\quad$ Non-intervention & NA & $6(11)$ \\
Surgical Outcome & & \\
Uncured & $6(13)$ & $6(11)$ \\
Cured & $27(57)$ & $27(51)$ \\
Open drainage & $14(30)$ & $14(26)$ \\
Tuberculosis Treatment Outcome & & \\
Cured & $21(45)$ & $23(43)$ \\
Under-treated & $16(34)$ & $19(36)$ \\
Treatment failure & $3(6)$ & $3(6)$ \\
Death & $3(6)$ & $4(8)$ \\
Unknown & $4(9)$ & $4(8)$ \\
Total & 47 & 53 \\
\hline NA $=$ not applicable & & \\
\hline
\end{tabular}

Twenty-three (43\%) of the pneumothorax patients were cured or completed their medical treatment. Three pneumothorax patients $(6 \%)$ had positive sputum smear at the end of the fifth month (treatment failure), 4 (8\%) died during medical TB treatment (from any cause). We could not obtain data on the treatment status of the other 4 patients. Nineteen pneumothorax patients $(36 \%)$ were under medical TB treatment during the study. Table 3 describes the surgeries and outcomes. The statistical analysis found no difference in medical TB treatment between the 2 groups.

\section{Discussion}

Contrary to the benign nature of primary spontaneous pneumothorax, pneumothorax secondary to an infection such as TB can be life-threatening because of the patient's underlying respiratory disease and the compromised cardiopulmonary reserve. ${ }^{16,17}$ In a study by Yagi et al, ${ }^{18}$ in Japan, 33\% of the TB patients with pneumothorax died. We did not compare our pneumothorax patients to non-TB patients with pneumothorax, to compare the frequency of clinical signs and symptoms and radiological manifestations. Instead, we compared our pneumothorax patients to a control group with confirmed TB patients, who were randomly selected from the registry of TB patients in our center. With that sample we could study clinical and radiological manifestations in predicting pneumothorax in TB patients.

In a study by Blanco-Perez et al, ${ }^{10}$ chest pain, cough, and fever were more frequent in patients with active TB 
than in non-TB patients with pneumothorax. On the other hand, our finding was that cough (85\%), dyspnea (70\%), and expectoration (70\%) were the most frequent symptoms. Pneumothorax was significantly more common in TB patients $<30$ years old $(P<.001)$; the mean age of our pneumothorax group was 34 years, which is closer to the age distribution of patients with primary spontaneous pneumothorax $(10-30$ y) than with secondary pneumothorax $(60-65 \mathrm{y})$, according to COPD population distribution. ${ }^{19}$ In contrast, previous studies found that other characteristics (eg, sex, smoking) were risk factors for primary pneumothorax. ${ }^{17}$ However, we found no association between those risk factors and TB-associated pneumothorax.

In $22(42 \%)$ of our patients the initial presentation at admission was for pneumothorax, and the TB was diagnosed thereafter. This indicates the importance of precise bacteriologic studies, including sputum smear for acid-fast bacilli, in patients who present with pneumothorax. In particular, when physicians encounter a remaining lesion after lung re-expansion following a spontaneous pneumothorax, they should consider pulmonary TB as a highly probable diagnosis. ${ }^{12}$ In the report by Yagi et al, ${ }^{18} 23$ patients (50\%) had pneumothorax as their first presentation.

In regard to radiological findings, there is a statistically significant association between cavitary lesions and pneumothorax. In our study, among the radiological manifestations, cavitary lesions on computed tomogram were significantly associated with pneumothorax. Among other radiological patterns, consolidation (40\%) and pulmonary infiltration $(36 \%)$ were the most frequent (see Table 2).

Of our 53 pneumothorax patients, 47 required surgery. All received a chest tube, oxygen supplementation, and close observation. Six patients (11\%) in whom thoracotomy was not performed were kept under close observation and were eventually cured by medical TB treatment, without surgery. Whether the patients who did not undergo surgery were kept under close observation is not clearly described in other reports, except for the report by Yagi et al, ${ }^{18}$ who reported that 11 patients $(24 \%)$ received aspiration and rest, 4 (9\%) underwent thoracentesis, 24 (52\%) had a chest tube inserted, and $7(15 \%)$ required further surgery. Those findings are consistent with our data. In those who underwent surgery, 27 (57\%) were cured, 14 $(30 \%)$ were followed with open drainage, and $6(13 \%)$ were not cured or did not show improvement. Only 3 patients $(6 \%)$ who received surgery died because of pneumothorax severity or recurrence (see Table 3 ). According to Blanco-Perez et al, ${ }^{10}$ despite the worse clinical condition of their patients with active TB (compared to their other patients), their active-TB patients had better surgery outcomes, though they did not mention exact figures.

We also assessed the TB treatment outcomes in our patients: an issue not addressed in previous reports. Of our
53 patients with TB and pneumothorax, 23 (43\%) completed medical TB treatment and were cured. Only $3(6 \%)$ of the patients had treatment failure. TB treatment outcome was not significantly different between our 2 groups (see Table 3).

\section{Limitations}

This study was conducted in a specialized referral center for TB and lung disease, so our results, particularly the morbidity and mortality, might not be representative of the general population of TB-induced pneumothorax patients. As there have been few studies of pneumothorax in TB patients, more comprehensive investigation should be undertaken.

We were surprised at the number of patients who first presented with pneumothorax and only subsequently were diagnosed with active TB. This finding highlights the necessity of performing bacteriologic studies, particularly sputum smear for acid-fast bacilli in patients who are hospitalized for pneumothorax.

\section{Conclusions}

The demographic, clinical, and radiologic findings in the patients were not characteristic for the diagnosis of pneumothorax in TB patients. However, considering the limitation of our small sample size, in younger patients with weight loss and cavitary lesions, worsening of respiratory condition should prompt consideration of pneumothorax, and performing early required management is recommended. Given the substantial morbidity and mortality of this clinically important condition, and especially in order to better understand risk factors and prognosis, further studies with larger sample sizes are crucial.

\section{REFERENCES}

1. Fox W. The chemotherapy of pulmonary tuberculosis: a review. Chest 1979;76(6 Suppl):785-796.

2. Mitchison DA. Treatment of tuberculosis: the Mitchell lecture 1979. J R Coll Physicians Lond 1980;14(2):91-95, 98-99.

3. Dye C, Williams BG. Eliminating human tuberculosis in the twentyfirst century. J R Soc Interface 2008;5(23):653-662.

4. Corbett EL, Watt CJ, Walker N, Maher D, Williams BG, Raviglione $\mathrm{MC}$, et al. The growing burden of tuberculosis: global trends and interactions with the HIV epidemic. Arch Intern Med 2003;163(9): 1009-1021.

5. Lucas SB, Hounnou A, Peacock C, Beaumel A, Djomand G, N'Gbichi JM, et al. The mortality and pathology of HIV infection in a west African city. AIDS 1993;7(12):1569-1579.

6. Mugusi FM, Mehta S, Villamor E, Urassa W, Saathoff E, Bosch RJ, et al. Factors associated with mortality in HIV-infected and uninfected patients with pulmonary tuberculosis. BMC Public Health 2009;9:409. 


\section{Tuberculosis-Associated Secondary Pneumothorax}

7. World Health Organization. Global tuberculosis control: surveillance, planning, financing. http://www.who.int/tb/publications/global_report/ 2006/en/index.html. Accessed December 21, 2010.

8. Treasure RL, Seaworth BJ. Current role of surgery in Mycobacterium tuberculosis. Ann Thorac Surg 1995;59(6):1405-1407; discussion 1408-1409.

9. Furak J, Trojan I, Szoke T, Tiszlavicz L, Morvay Z, Csada E, et al. Surgical intervention for pulmonary tuberculosis: analysis of indications and perioperative data relating to diagnostic and therapeutic resections. Eur J Cardiothorac Surg 2001;20(4):722-727.

10. Blanco-Perez J, Bordón J, Piñeiro-Amigo L, Roca-Serrano R, Izquierdo R, Abal-Arca J. Pneumothorax in active pulmonary tuberculosis: resurgence of an old complication? Respir Med 1998;92(11): 1269-1273.

11. Olcmen A, Gunluoglu MZ, Demir A, Akin H, Kara HV, Dincer SI. Role and outcome of surgery for pulmonary tuberculosis. Asian Cardiovasc Thorac Ann 2006;14(5):363-366.

12. Kim HY, Song KS, Goo JM, Lee JS, Lee KS, Lim TH. Thoracic sequelae and complications of tuberculosis. Radiographics 2001; 21(4):839-858; discussion 859-860.
13. Ihm HJ, Hankins JR, Miller JE, McLaughlin JS. Pneumothorax associated with pulmonary tuberculosis. J Thorac Cardiovasc Surg 1972;64(2):211-219.

14. Belmonte R, Crowe HM. Pneumothorax in patients with pulmonary tuberculosis. Clin Infect Dis 1995;20(6):1565.

15. World Health Organization. Guidelines for the control of TB. http:// www.wpro.who.int/NR/rdonlyres/373982C6-3485-4AA8-896F10C1907D366E/0/tbguide.pdf.

16. Lim KH, Tan LH, Liam CK, Wong CM. An unusual cause of secondary spontaneous pneumothorax in a 27 -year-old man. Chest 2001 ; 120(5):1728-1731.

17. Sahn SA, Heffner JE. Spontaneous pneumothorax. N Engl J Med 2000;342(12):868-874.

18. Yagi T, Yamagishi F, Sasaki Y, Mizutani F, Wada A, Kuroda F. [Clinical review of pneumothorax cases complicated with active pulmonary tuberculosis]. Kekkaku 2002;77(5):395-399. Article in Japanese.

19. Primrose WR. Spontaneous pneumothorax: a retrospective review of aetiology, pathogenesis and management. Scott Med J 1984;29(1): $15-20$. 